\title{
Average Real Variability of Diastolic Blood Pressure During Acute Phase Could Independently Predict 3-month Functional Outcome for Ischemic Stroke
}

\author{
Wenzhu Liu ${ }^{1}$, Yanping Chen ${ }^{1}$, Xiafeng $\mathrm{Wu}^{2}$, Zefeng $\operatorname{Tan}^{3,}$ * \\ ${ }^{1}$ South Campus Outpatient Department, The First Affiliated Hospital, Jinan University, Guangzhou, China \\ ${ }^{2}$ Department of Haematology, The First Affiliated Hospital of Jinan University, Guangzhou, China \\ ${ }^{3}$ Department of Neurology, The First Affiliated Hospital of Jinan University, Guangzhou, China
}

Email address:

8190804@qq.com (Zefeng Tan)

${ }^{*}$ Corresponding author

\section{To cite this article:}

Wenzhu Liu, Yanping Chen, Xiafeng Wu, Zefeng Tan. Average Real Variability of Diastolic Blood Pressure During Acute Phase Could Independently Predict 3-month Functional Outcome for Ischemic Stroke. American Journal of Internal Medicine.

Vol. 8, No. 3, 2020, pp. 107-112. doi: 10.11648/j.ajim.20200803.13

Received: March 29, 2020; Accepted: April 15, 2020; Published: April 30, 2020

\begin{abstract}
Background and purpose-Blood pressure variability has a significant effect on stroke. There were controversial reports about its relationship with short term functional outcome remains controversial for ischemic stroke or transient ischemic attack (TIA). There were limited studies about its long-term effect. Our aim was to elucidate the effect of BPV on a 3-month functional outcome in patients with acute ischemic stroke or TIA. Methods - In this prospective observational study, a consecutive series of 400 patients were enrolled with acute ischemic stroke or TIA within 7 days. All patients were referred and monitored for BP at 2-hour intervals in the first 24 hours. Afterward, the BP was recorded every four hours up to the 7th day, with 1-hour deviation the daytime and 2 hours at night. All BP results were recorded into an electronic medical record (EMR) system. Average real variability (ARV) was used to analyze the blood pressure variability (BPV). All patients were followed up as planned for 90 days. The primary outcome was evaluated by the modified Rankin Scale (mRS); an unfavorable outcome was $\mathrm{mRS} \geq 2$. For comparison, patients were respectively divided into two groups based on systolic blood pressure ARV (SBP ARV) or diastolic blood pressure ARV (DBP ARV). A multivariate logistic regression model was used to estimate the effect of between ARV BPV on functional outcomes. Results-Among the 400 patients, 46 (11.4\%) had an unfavorable outcome $(\mathrm{mRS}>2)$ at 90 days. Their mean 24h DBP ARV was significantly higher than others $(10.42 \pm 3.63$ vs. $9.83 \pm 4.27 \mathrm{~mm} \mathrm{Hg}$; $\mathrm{p}=0.03$ ). The high $24 \mathrm{hDBPARV}$ was defined above the median of $7.75 \mathrm{mmHg}$, which was statistically associated with an unfavorable outcome $(64.7 \%$ vs. $35.3 \%$; $\mathrm{P}=0.004)$. Further stepwise logistic regression analysis indicated that $24 \mathrm{~h}$ high DBPARV was the independent predictor of an unfavorable outcome (adjusted OR 2.44\%, 95\% confidence interval: 1.24-4.74, $\mathrm{P}<0.01$ ). Conclusions - High DBPARV during the acute phase is an independent and significant predictor of 90-day functional outcome for ischemic stroke or TIA.
\end{abstract}

Keywords: Blood Pressure in Acute Stroke, Ischemic Stroke, Transient Ischemic Attack, Acute Stroke Outcome

\section{Introduction}

Blood pressure variation (BPV) - the variation of blood pressure continuously with time [1-3] has been proposed as a reliable and independent predictor of the long-term risk of stroke, which was more specific for prediction than simple blood pressure levels [4]. The clinical significance of BPV is uncertain during the acute phase of the stroke. A recent systematic review of seven studies suggested that systolic BPV was significantly associated with poor functional outcomes (pooled odds ratio per $10 \mathrm{~mm} \mathrm{Hg}$ increment, 1.2; 95\% confidence interval: 1.1-1.3) [5]. More prospective studies were required to investigate how best to measure and define the BPV standard in acute stroke, as well as to determine its prognostic significance [5]. Various mathematical indexes were used to evaluate BPV in 
different reports, such as standard deviation (SD), coefficient of variation $(\mathrm{CV})$, variation independent of the mean (VIM), weighted standard deviation (wSD), and average real variability (ARV). But It's also uncertain for variability index calculation to precisely demonstrate the BPV significance for the prognostic prediction. This observational study was conducted to investigate the correlation between the BPARV and the short-term functional outcome in patients with acute ischemic stroke or transient ischemic attack (TIA).

\section{Methods}

\subsection{Subjects and Setting}

This was a prospective observational study, including 451 patients with ischemic stroke or TIA within 7 days after onset. They were admitted to the Department of Neurology, the First Affiliated Hospital, Jinan University, Guangzhou, China, from August 2012 to August 2013. All patients were monitored in the neurological intensive care unit (NICU) in the first 24 hours after admission. Afterward, patients were transferred out NICU according to its performance and clinical diagnosis. Cranial computerized tomography (CT) or magnetic resonance imaging (MRI) and magnetic resonance angiography (MRA) were performed routinely within 24 hours after admission. TIA and ischemic stroke were diagnosed according to standardized diagnostic criteria [6]. This study excluded cases with thrombolysis, inadequate BP measurements, other life-threatening comorbidities before stroke onset, or modified Rankin Scale score $(\mathrm{mRS})>2$ caused by a previous stroke. The research protocol was approved by the medical ethical committee of the First Affiliated Hospital, Jinan University, for clinical research, and informed consent was obtained from all participants.

\subsection{Measurement of Blood Pressure}

Casual supine blood pressure (BP) was measured in the non-paralyzed arm using a standard electronic sphygmomanometer (OMROM-HEM-7200) and the appropriate cuff size on admission. BP was measured at 2hour intervals throughout the first 24 hours using a noninvasive BP monitoring device (Bene View T5) in NICU, and then every 4 hours, up to the 7 th day in general ward after admission, using a standard electronic sphygmomanometer (OMROM-HEM-7200) by trained nurses, with 1-hour deviation in the day and 2 hours at night. All BP results were recorded into the electronic medical record (EMR) system. The BP profile during the first 24 hours $(\mathrm{h})$ and the 2-7 days $\left(\mathrm{D}_{2-7}\right)$ was described using various parameters for each of systolic blood pressure (SBP) and diastolic blood pressure (DBP): SD, CV. As an index of short term BPV, average real variability (ARV) [7] was used for evaluation, which was weighted for the time interval between consecutive readings. It was calculated by the following formula:

$$
\mathrm{ARV}=\frac{1}{\mathrm{~N}-1} \sum_{\mathrm{k}=1}^{\mathrm{n}-1} \times\left|\mathrm{BP}_{\mathrm{K}+1}-\mathrm{BP}_{\mathrm{K}}\right|
$$

$\mathrm{k}$ ranges from 1 to $\mathrm{n}-1$ and $\mathrm{w}$ is the time interval between $\mathrm{BP}_{\mathrm{k}}$ and $\mathrm{BP}_{\mathrm{k}+1} . \mathrm{n}$ is the number of blood pressure readings.

\subsection{Stroke Risk Factors}

The prognostic functional outcome at 90days and the baseline National Institute of Health stroke scale (NIHSS) score were collected as planned. Other general personal characteristics were also recorded, including age, gender, hypertension ( $\mathrm{BP} \geq 140 / 90 \mathrm{mmHg}$ [8] or antihypertensive treatment), diabetes mellitus, hyperlipidemia, symptomatic ischemic heart disease (myocardial infarction, angina or existence of multiple lesions on thallium heart isotope screen or coronary disease), atrial fibrillation, current cigarette smoking, alcohol abuse ( $>50 \mathrm{~g}$ per week), and stroke history.

\subsection{Assessment of the Clinical Outcome}

Two trained stroke neurologists were blinded and respectively arranged to assess functional outcome at 90 days. The functional outcome was assessed by the mRS score at $90 \pm 7$ days after the treatment. It was dichotomized into a favorable outcome (mRS 0 to 1 ) or unfavorable outcome (mRS 2 to 6 , death was graded 6). On admission ( $1^{\text {st }}$ day) and on the $90^{\text {th }}$ day, the modified ranking scale was evaluated.

\subsection{Statistical Analysis}

All analysis was performed using SPSS for Windows, release 19.0 (SPSS Inc., Chicago, IL, USA), and $\mathrm{P}<0.05$ was considered as statistically significant. Quantitative variables were summarized as mean $\pm \mathrm{SD}$, categorical variables as frequency distribution. Comparisons of baseline characteristics or BPV parameters for predicting functional outcome were conducted with the Pearson $\chi^{2}$ test, MannWhitney $\mathrm{U}$ test, or Student $t$-test as described. For ARV analysis, patients were divided into low ( $\leq$ median) and high (>median) groups using the median value of systolic and diastolic ARV during the first 24 hours and over the subsequent 2-7 days. The patient proportion with the unfavorable outcome was calculated and its correlation with ARV was assessed using a Pearson $\chi^{2}$ test. All parameters with statistical significance $(\mathrm{p}<0.05)$ in the univariate analysis were introduced into a multivariate logistic regression model to explore predictors for unfavorable functional outcome. Due to the possible collinearity of tested variables, a stepwise logistic regression model was used.

\section{Results}

451patients were initially enrolled with ischemic stroke or TIA. Fifty-one patients were excluded without the follow-up response. For the final 400 patients from 2012-2013, the median age was $64.3 \pm 10.3$ (range 34-93) years, and 123 patients were female. At admission, 372 patients (93\%) were diagnosed with ischemic stroke, and 28 patients $(7 \%)$ had TIA. A history of hypertension was present in 247 (61.8\%) 
patients. Most patients did not know their typical blood pressure values. $58.1 \%$ were under antihypertensive therapy. All patients were given standardized therapy according to the guidelines. Forty-four patients (11\%) were given antihypertension therapy, 386 patients $(96.5 \%)$ antiplatelet therapy, and 385 patients $(96.2 \%)$ were prescribed with statin therapy. Among them, 208 cases were minor stroke $($ NIHSS $<4)$. Demographic data and clinical characteristics of 400 patients were presented in Table 1 . Admission within 12 hours after onset was significantly associated with better functional outcome $(P=0.018)$.

Table 1. Comparison of clinical data between the favorable and unfavorable outcomes groups patients.

\begin{tabular}{|c|c|c|c|}
\hline Patient characteristics & $\begin{array}{l}\text { Favorable outcomes (mRS 0-2) } \\
(\mathrm{n}=354)\end{array}$ & $\begin{array}{l}\text { Unfavorable outcomes (mRS 3-5) } \\
(\mathrm{n}=46)\end{array}$ & $P$ value \\
\hline Female (\%) & $108(30.5 \%)$ & $15(32.6 \%)$ & 0.772 \\
\hline Age (years) & $63.5 \pm 13.0$ & $65.2 \pm 14.3$ & 0.429 \\
\hline Current smoker (\%) & $119(33.6 \%)$ & $14(30.4 \%)$ & 0.667 \\
\hline Alcohol consumption $(\%)$ & $28(7.9 \%)$ & $3(6.5 \%)$ & 0.853 \\
\hline Previous stroke (\%) & $76(21.4 \%)$ & $9(19.6 \%)$ & 0.767 \\
\hline Coronary artery disease (\%) & $43(12.1 \%)$ & $6(13.0 \%)$ & 0.861 \\
\hline Hyperlipidemia (\%) & $18(5.0 \%)$ & $4(8.7 \%)$ & 0.505 \\
\hline Atrial fibrillation (\%) & $23(6.5 \%)$ & $5(10.9 \%)$ & 0.274 \\
\hline Diabetes mellitus (\%) & $90(25.4 \%)$ & $8(17.4 \%)$ & 0.233 \\
\hline History of hypertension (\%) & $223(62.9 \%)$ & $24(52.2 \%)$ & 0.148 \\
\hline Interval time for onset to admission $\leq 12$ hours $(\%)$ & $162(45.8 \%)$ & $12(26.1 \%)$ & 0.018 \\
\hline TIA $(\%)$ & $28(7.9 \%)$ & $0(0 \%)$ & 0.048 \\
\hline Antihypertension therapy (\%) & $40(11.3 \%)$ & $4(5.8 \%)$ & 0.595 \\
\hline Antiplatelet therapy (\%) & $343(98.0 \%)$ & $43(93.4 \%)$ & 0.383 \\
\hline Statin therapy (\%) & $340(96.0 \%)$ & $45(97.8 \%)$ & 0.416 \\
\hline NIHSS at admission, Median (IQR) & $3(1,7)$ & $1(1,1)$ & 0.137 \\
\hline \multicolumn{4}{|l|}{ Blood pressure at admission $(\mathrm{mmHg})$} \\
\hline SBP & $155.2 \pm 24.7$ & $153.8 \pm 24.0$ & 0.710 \\
\hline DBP & $86.7 \pm 15.8$ & $86.5 \pm 20.7$ & 0.948 \\
\hline $\mathrm{TC}^{*}(\mathrm{mmol} / \mathrm{L})$ & $4.9 \pm 1.2$ & $5.7 \pm 4.6$ & 0.299 \\
\hline $\mathrm{TG}(\mathrm{mmol} / \mathrm{L})$ & $1.8 \pm 1.2$ & $1.7 \pm 1.2$ & 0.540 \\
\hline LDL-C (mmol/L) & $3.1 \pm 1.5$ & $2.9 \pm 1.0$ & 0.589 \\
\hline HDL-C (mmol/L) & $1.2 \pm 0.7$ & $1.2 \pm 0.3$ & 0.579 \\
\hline $\mathrm{FBG}(\mathrm{mmol} / \mathrm{L})$ & $6.7 \pm 3.0$ & $6.2 \pm 2.2$ & 0.320 \\
\hline $\mathrm{HbA1c}$ & $6.5 \pm 1.9$ & $6.3 \pm 1.5$ & 0.554 \\
\hline D-D & $1216.3 \pm 1934.2$ & $1278.6 \pm 1639.8$ & 0.840 \\
\hline hs-CRP (mg/L) & $14.5 \pm 37.8$ & $12.5 \pm 26.3$ & 0.745 \\
\hline
\end{tabular}

Comparisons of systolic and diastolic BPV parameters between favorable and unfavorable outcome patients were presented in Table 2. Unfavorable results were significantly associated with higher $24 \mathrm{~h}$ DBP ARV $(P=0.03)$. For the univariate analysis of systolic or diastolic ARV, patients were respectively divided into groups with low and high ARV. The significant association was demonstrated between high 24hDBP-ARV and unfavorable functional outcome $(P=0.005)$ (Table 3). After adjusting the known baseline predictors, 24h DBP ARV, and admission within 12 hours after onset were associated with favorable outcome at day 90 (Table 4).

Table 2. Comparison of Systolic and Diastolic BPV Parameters between the favorable and unfavorable outcome groups patients.

\begin{tabular}{|c|c|c|c|}
\hline & Favorable outcomes $(n=354)$ & Unfavorable outcomes $(n=46)$ & $P$ Value \\
\hline 24h SBP-SD $(\mathrm{mmHg})^{*}$ & $13.1 \pm 5.3$ & $12.9 \pm 5.6$ & 0.826 \\
\hline 24h SBP-CV* & $0.1 \pm 0.0$ & $0.1 \pm 0.0$ & 0.997 \\
\hline 24h SBP-ARV (mmHg) & $11.2 \pm 4.6$ & $10.5 \pm 4.1$ & 0.300 \\
\hline 24h DBP-SD (mmHg) & $10.8 \pm 5.3$ & $11.8 \pm 5.6$ & 0.269 \\
\hline 24h DBP-CV & $0.2 \pm 0.1$ & $0.2 \pm 0.1$ & 0.377 \\
\hline 24h DBP-ARV (mmHg) & $9.8 \pm 4.3$ & $10.4 \pm 3.6$ & 0.030 \\
\hline $\mathrm{D}_{2-7} \mathrm{SBP}-\mathrm{SD}(\mathrm{mmHg})^{*}$ & $12.8 \pm 4.7$ & $13.0 \pm 4.3$ & 0.778 \\
\hline $\mathrm{D}_{2-7} \mathrm{SBP}-\mathrm{ARV}(\mathrm{mmHg})^{*}$ & $12.0 \pm 4.6$ & $12.4 \pm 4.0$ & 0.579 \\
\hline $\mathrm{D}_{2-7} \mathrm{DBP}-\mathrm{SD}(\mathrm{mmHg})^{*}$ & $8.2 \pm 3.1$ & $8.7 \pm 2.9$ & 0.288 \\
\hline $\mathrm{D}_{2-7}$ DBP-CV & $0.1 \pm 0.0$ & $0.1 \pm 0.0$ & 0.356 \\
\hline $\mathrm{D}_{2-7}$ DBP-ARV (mmHg) & $8.1 \pm 3.2$ & $8.5 \pm 2.6$ & 0.433 \\
\hline
\end{tabular}

Values are mean \pm SD. SBP, systolic blood pressure; DBP, diastolic blood pressure; CV, coefficient of variation; ARV, average real variability. 
Table 3. Univariate analysis of patients with high and low systolic and diastolic ARV between patients of favorable and unfavorable outcomes groups.

\begin{tabular}{lccl}
\hline & Favorable outcomes $(\mathbf{n}=\mathbf{3 5 4})$ & Unfavorable outcomes $(\mathbf{n}=\mathbf{4 6})$ & P Value \\
\hline 24h SBP-ARV & & & 0.555 \\
Low $(\leq 11.0)$ & $176(49.7)$ & $25(54.3)$ & \\
High $(>11.0)$ & $178(50.3)$ & $21(45.7)$ & 0.004 \\
24h DBP-ARV & & $13(28.2)$ & \\
Low $(\leq 7.8)$ & $187(52.8)$ & $33(71.7)$ & 0.531 \\
High (>7.8) & $167(47.2)$ & & \\
D $_{2-7}$ SBP-ARV & & $25(54.3)$ & 0.508 \\
Low $(\leq 11.8)$ & $175(49.4)$ & $21(45.7)$ & \\
High $(>11.8)$ & $179(50.6)$ & $21(45.7)$ & \\
D $_{2-7}$ DBP-ARV & & $25(54.3)$ & \\
Low $(\geq 7.8)$ & $180(50.8)$ & & \\
High $(>7.8)$ & $174(49.2)$ & & \\
\hline
\end{tabular}

Table 4. Final stepwise logistic regression model to predict unfavorable outcomes.

\begin{tabular}{lll}
\hline Variable & Adjusted OR (95\%CI) & PValue \\
\hline Interval time $<$ 12hours & $0.45(0.23,0.91)$ & 0.009 \\
24h high DBP-ARV & $2.43(1.24,4.74)$ & 0.001 \\
\hline
\end{tabular}

SBP, systolic blood pressure; ARV, average real variability.

(Final stepwise logistic regression model to predict poor outcome at 90 days, included variables: interval time from onset to admission, and 24hours DBPARV).

\section{Discussion}

Blood pressure variability might significantly predict the prognostic outcome of clinical stroke. In previous studies [1116], BP monitoring was recommended at the early stage of stroke. In acute ischemic stroke, dramatic short-term change in BP might significantly influence the patients' clinical performance and follow-up outcome. Previous reports [17-20] showed that BPV could effectively predict the long-term functional outcome, and SBPV was independently associated with a long-term unfavorable functional outcome [11-16, 2123]. There was a limited prospective study about the clinical significance of BPV in the short-term functional outcome in the acute stage of ischemic stroke.

Our research protocol was designed to investigate the BPV prediction for acute functional outcomes. As expected, higher DBP ARV in the first 24 hours was found as the predictor for a 3-month unfavorable functional outcome. As reported, there were only 8 studies about the association between DBPV and stroke outcome [23]. Among them, only one study confirmed DBPV was related to the outcome at 30 days [24]. But these studies enrolled patients with intracerebral hemorrhage and ischemic stroke simultaneously, and results were biased due to the mixed sample. More importantly, varied BPV parameters were used to analyze different outcomes [5]. Instead, we solely collected ischemic stroke patients and introduced one unique parameter, ARV BPV, to discuss the clinical correlation between DBPV and outcome at 90 days. Currently, there was no consensus parameter to estimate BPV. Various indexes were applied in previous studies, including SD squared differences, CV, wSD, variation independent of mean (transformation of SD uncorrelated with mean), and so on. The effects of DBPV indices on outcome were considered in 16 studies. SD DBP was the most frequent parameter [5]. For accurate BPV assessment, SD and CV ignore the data order of BP, and two sets with different clinical meanings might have the same SD or CV. wSD ignores the adverse effect of nocturnal decreases in BP, while VIM is not suitable for clinical application ${ }^{7}$. ARV included more information about data variance and its sequence order. ARV adequately takes consideration of the sequential effect of BP monitoring instead of simple value variance. It might reflect the individual's BPV more accurately. ARV is also reliable and straightforward to be calculated and easy for clinical practice.

In our results, the mean $24 \mathrm{~h}$ DBP ARV at baseline was significantly higher among patients with an unfavorable outcome than others at 90 days $(10.42 \pm 3.63$ vs. $9.83 \pm 4.27 \mathrm{~mm} \mathrm{Hg} ; p=0.03)$. The high $24 \mathrm{hDBPARV}$, above the median of $7.75 \mathrm{mmHg}$, was definitely associated with an unfavorable outcome $(64.7 \%$ vs. $35.3 \% ; P=0.004)$. Further stepwise logistic regression analysis showed that $24 \mathrm{~h}$ high DBPARV was the strong and independent predictor of an unfavorable outcome (adjusted OR 2.44\%, 95\% confidence interval: $1.24-4.74, P<0.01$ ).

The detailed mechanism for our finding was unknown. Normally, systolic and diastolic BP will parallel change under physiological stimulation. This paralleling correlation might be altered due to stiff arteries [25-26]. SBP increases, but DBP increases less or even decrease, which could cause a more substantial variability. The pathophysiological effects of more extensive DBPVon brain tissue might vary based on the impairment degree in cerebral auto-regulation, which is influenced by infarct size and potentially salvageable penumbral tissue [27-28]. Perhaps in the initial period after onset, the potentially salvageable penumbral tissue was particularly vulnerable to BP fluctuation, where acute BP declines, especially DBP, increased the ischemia risk and reduced the reperfusion opportunity. This also explained why there was no significance between DBPV after 12 hours and short-term functional outcome in this study. This speculated mechanism also was referred to us by other large-scale studies, such as ECASSI and ECASSII [16]. The ECASSI study showed that DBPV decreases within 3 days after admission was independently related to a better functional outcome. In the ECASSII study [16], DBPV within 24 hours after admission was related to less hemorrhagic transformation and better functional outcomes. 
There were very seldom prospective studies that applied one variability parameter as a clinical predictor for the stroke functional outcome [14]. Our DBP ARV result might significantly affect clinical practice for treating ischemic stroke or TIA patients. Our study still had limitations and need further verification. The large cohort study in multiple centers should be performed instead of our single hospital. Instead of minor stroke cases, more severe patients should be recruited to explore the clinical significance of DBP ARV in the stroke population. With the increasing cohort, the stratification analysis was applied to verify the clinical correlation between short-term functional outcomes of minor ischemic strokes to the BPV. The potential mechanism should also be studied biologically and clinically.

\section{Conclusion}

Our research implied that increased DBP ARV within the first 24 hours had impacts on functional recovery in acute ischemic stroke or TIA patients. These findings demonstrated the importance of BP monitoring and BP controlling in the acute phase of ischemic stroke. Further large scale multicenter prospected studies were required to verify our findings and explore the possible underlying mechanism.

\section{Sources of Funding}

This work was supported by the Tianhe district science and technology project 2018YT006, China.

\section{Acknowledgements}

We thank all the neurologists and nurses in the Department of Neurology and outpatient department, the First Affiliated Hospital of Jinan University.

\section{References}

[1] Mancia G, Grassi G. Mechanisms and clinical implications of blood pressure variability. J Cardiovasc Pharmacol. 2000; 35: S15-19.

[2] Ohkubo T. Prognostic significance of variability in ambulatory and home blood pressure from the Ohasama study. $J$ Epidemiol. 2007; 17: 109-113.

[3] Miao CY, Xie HH, Zhan LS, Su DF. Blood pressure variability is more important than blood pressure level in determination of end-organ damage in rats. J Hypertens. 2006; 24: 11251135 .

[4] Rothwell PM, Howard SC, Dolan E, O'Brien E, Dobson JE, Dahlöf B, Sever PS, Poulter NR. Prognostic significance of visit-to-visit variability, maximum systolic blood pressure, and episodic hypertension. Lancet. 2010; 375: 895-905.

[5] Manning LS, Rothwell PM, Potter JF, Robinson TG. Prognostic Significance of Short-Term Blood Pressure Variability in Acute Stroke: Systematic Review. Stroke. 2015; 46: $2482-2490$.
[6] Sacco RL, Kasner SE, Broderick JP, Caplan LR, Connors JJ, Culebras A, Elkind MS, George MG, Hamdan AD, Higashida RT, Hoh BL, Janis LS, Kase CS, Kleindorfer DO, Lee JM, Moseley ME, Peterson ED, Turan TN, Valderrama AL, Vinters HV. An updated definition of stroke for the 21st century: a statement for healthcare professionals from the American Heart Association/American Stroke Association. Stroke. 2013; 44: 2064-2089.

[7] Mena L, Pintos S, Queipo NV, Aizpúrua JA, Maestre G, Sulbarán T. A reliable index for the prognostic significance of blood pressure variability. J Hypertens. 2005; 23: 505-511.

[8] 1999 World Health Organization-International Society of Hypertension Guidelines for the Management of Hypertension. Guidelines Subcommittee. J Hypertens. 1999; 17: 151-183.

[9] Alberti KG, Zimmet PZ. Definition, diagnosis and classification of diabetes mellitus and its complications. Part 1: diagnosis and classification of diabetes mellitus provisional report of a WHO consultation. Diabet Med. 1998; 15: 539553.

[10] Standards of medical care in diabetes--2011. Diabetes Care. 2011; 34 Suppl 1: S11-61.

[11] Delgado-Mederos R, Ribo M, Rovira A, Rubiera M, Munuera J, Santamarina E, Delgado P, Maisterra O, Alvarez-Sabin J, Molina CA. Prognostic significance of blood pressure variability after thrombolysis in acute stroke. Neurology. 2008; 71: 552-558.

[12] Endo K, Kario K, Koga M, Nakagawara J, Shiokawa Y, Yamagami H, Furui E, Kimura K, Hasegawa Y, Okada Y, Okuda S, Namekawa M, Miyagi T, Osaki M, Minematsu K, Toyoda K. Impact of early blood pressure variability on stroke outcomes after thrombolysis: the SAMURAI rt-PA Registry. Stroke. 2013; 44: 816-818.

[13] Kellert L, Sykora M, Gumbinger C, Herrmann O, Ringleb PA. Blood pressure variability after intravenous thrombolysis in acute stroke does not predict intracerebral hemorrhage but poor outcome. Cerebrovasc Dis. 2012; 33: 135-140.

[14] Manning L, Hirakawa Y, Arima H, Wang X, Chalmers J, Wang J, Lindley R, Heeley E, Delcourt C, Neal B, Lavados P, Davis SM, Tzourio C, Huang Y, Stapf C, Woodward M, Rothwell PM, Robinson TG, Anderson CS. Blood pressure variability and outcome after acute intracerebral haemorrhage: a post-hoc analysis of INTERACT2, a randomised controlled trial. Lancet Neurol. 2014; 13: 364-373.

[15] Sare GM, Ali M, Shuaib A, Bath PM. Relationship between hyperacute blood pressure and outcome after ischemic stroke: data from the VISTA collaboration. Stroke. 2009; 40: 2098-2103.

[16] Yong M, Kaste M. Association of characteristics of blood pressure profiles and stroke outcomes in the ECASS-II trial. Stroke. 2008; 39: 366-372.

[17] Webb AJ, Fischer U, Mehta Z, Rothwell PM. Effects of antihypertensive-drug class on interindividual variation in blood pressure and risk of stroke: a systematic review and meta-analysis. Lancet. 2010; 375: 906-915.

[18] Rothwell PM, Howard SC, Dolan E, O'Brien E, Dobson JE, Dahlöf B, Poulter NR, Sever PS. Effects of beta blockers and calcium-channel blockers on within-individual variability in blood pressure and risk of stroke. Lancet Neurol. 2010; 9: 469-480. 
[19] Moher D, Liberati A, Tetzlaff J, Altman DG. Preferred reporting items for systematic reviews and meta-analyses: the PRISMA statement. Int J Surg. 2010; 8: 336-341.

[20] Stroup DF, Berlin JA, Morton SC, Olkin I, Williamson GD, Rennie D, Moher D, Becker BJ, Sipe TA, Thacker SB. Metaanalysis of observational studies in epidemiology: a proposal for reporting. Meta-analysis Of Observational Studies in Epidemiology (MOOSE) group. JAMA. 2000; 283: 20082012.

[21] Tanaka E, Koga M, Kobayashi J, Kario K, Kamiyama K, Furui E, Shiokawa Y, Hasegawa Y, Okuda S, Todo K, Kimura $\mathrm{K}$, Okada Y, Okata T, Arihiro S, Sato S, Yamagami $\mathrm{H}$, Nagatsuka K, Minematsu K, Toyoda K. Blood pressure variability on antihypertensive therapy in acute intracerebral hemorrhage: the Stroke Acute Management with Urgent Riskfactor Assessment and Improvement-intracerebral hemorrhage study. Stroke. 2014; 45: 2275-2279..

[22] Kang J, Ko Y, Park JH, Kim WJ, Jang MS, Yang MH, Lee J, Lee J, Han MK, Gorelick PB, Bae HJ. Effect of blood pressure on 3-month functional outcome in the subacute stage of ischemic stroke. Neurology. 2012; 79: 2018-2024.

[23] Manning LS, Mistri AK, Potter J, Rothwell PM, Robinson TG. Short-term blood pressure variability in acute stroke: post hoc analysis of the controlling hypertension and hypotension immediately post stroke and continue or stop post-stroke antihypertensives collaborative study trials. Stroke. 2015; 46: 1518-1524.

[24] Dawson SL, Manktelow BN, Robinson TG, Panerai RB, Potter JF. Which parameters of beat-to-beat blood pressure and variability best predict early outcome after acute ischemic stroke. Stroke. 2000; 31: 463-468.

[25] Li Y, Wang JG, Dolan E, Gao PJ, Guo HF, Nawrot T, Stanton AV, Zhu DL, O'Brien E, Staessen JA. Ambulatory arterial stiffness index derived from 24-hour ambulatory blood pressure monitoring. Hypertension. 2006; 47: 359-364.

[26] Macwilliam JA, Melvin GS. Systolic and diastolic blood pressure estimation, with special reference to the auditory method. Br Med J. 1914; 1: 693-697.

[27] Reinhard M, Rutsch S, Lambeck J, Wihler C, Czosnyka M, Weiller C, Hetzel A. Dynamic cerebral autoregulation associates with infarct size and outcome after ischemic stroke. Acta Neurol Scand. 2012; 125: 156-162.

[28] Guo ZN, Liu J, Xing Y, Yan S, Lv C, Jin H, Yang Y. Dynamic cerebral autoregulation is heterogeneous in different subtypes of acute ischemic stroke. PLoS One. 2014; 9: e93213. 